\title{
Danuta Rytel-Schwarz / Alina Jurasz / Lesław Cirko / Ulrich Engel (2018), Deutsch-polnische kontrastive Grammatik. Bd. 2: Der Satz, 2., neu bearbeitete und ergänzte Auflage. Hildesheim/Zürich/New York: Olms Verlag, 462 S., ISBN 978-3-487-14783-3.
}

DOI: $10.19195 / 0137-1169.38 .9$

Die erste, zweibändige Ausgabe der „Deutsch-polnischen kontrastiven Grammatik” ist vor zwanzig Jahren erschienen und war Gegenstand vieler Diskussionen. Es gab Rezensionen (z.B. Lipczuk 2000, Nagórko 2000), zerstreute Aufsätze (z.B. Feret 2010) und vor allem zwei internationale Linguistenkonferenzen (Karpacz 2006 und 2007), deren Teilnehmer sich ausschließlich mit diversen Aspekten des genannten Nachschlagewerks auseinandergesetzt haben. Die Vorträge sind in den Sammelbänden „Zwischen Lob und Kritik: sechs Jahre Erfahrung mit der Deutsch-polnischen Grammatik (dpg)“ und „DPG im Kreuzfeuer“ veröffentlicht worden (vgl. Cirko et al. 2008, 2009). Dies alles zeugt sowohl vom regen Interesse als auch vom großen Bedarf an einer solchen Grammatik.

Die Publikation war das Ergebnis intensiver Zusammenarbeit polnischer und deutscher Germanisten und Polonisten. Nach Jahren beschlossen einige Autoren, dem Gedanken folgend, dass „die Erstauflage teilweise nicht mehr den theoretischen und didaktischen Anforderungen an ein Werk (entspricht), das auch im modernen universitären Grammatikunterricht einsetzbar ist" (Vorwort, S. 13), die Grammatik neu zu bearbeiten. Die zu modifizierenden und zu aktualisierenden Aspekte werden in der neuen Ausgabe allgemein genannt: „Die Kritiker der ersten Auflage hatten auf etliche Inkongruenzen in der Gegenüberstellung beider Sprachsysteme aufmerksam gemacht, vor allem auf die nicht immer befriedigende Beschreibung der polnischen Seite und die nicht konsequent genug gehaltene kontrastive Darstellung. Neuere Forschungsergebnisse der Polonistik waren miteinzubeziehen. Im Zusammenhang mit weiteren kontrastiven Projekten waren gewisse Veränderungen der zugrundeliegenden Theorie - der Dependenziellen Verbgrammatik (DVG) - zu berücksichtigen“ (ebd., S. 13-14).

Von den vier Bänden der überarbeiteten und erweiterten Ausgabe sind 2012 Band 4: „Die unflektierbaren Wörter“ und 2018 Band 2: „Der Satz“ erschienen. Die weiteren Bände: „Die flektierbaren Wörter. Das Verb. Der nominale Bereich“ und „Text. Phonetik. Schichtübergreifendes“ sind noch in Vorbereitung.

Der neueste Band besteht aus fünf Hauptkapiteln: 1. Allgemeines - wo u.a. die Klassifizierung der Wörter in Wortklassen, Dependenzrelationen, Rektion, Valenz und Satzstrukturen besprochen werden; 2. Grundbegriffe - dieser Teil führt den Leser in die Problematik des Satzes, seiner Komponenten und Typen ein; 3. Der einfache Satz - mit mehreren Unterkapiteln zu den einzelnen Ergänzungs- und Angabentypen sowie zu Satzmustern; 4. Der komplexe Satz - hier werden die Verknüpfungsmittel, Ergänzungs-Komplexe, Angabesatz-Komplexe, Attributsatz-Komplexe und Satzreihen dargestellt; und 5. Stellungsregeln - mit separaten Teilen über Satzrahmen und Stellungsfelder, die Stellung im Verbalkomplex, Grundfolge der Ergänzungen und Angaben, Permutationen und die Stellung satzartiger Konstruktionen. Die Gliederung ist übersichtlicher und benutzerfreundlicher als in der Ausgabe von 1999. Nicht alle Kapitel der ersten Ausgabe sind hier als Kapitel berücksichtigt 
worden. So sind z.B. die separaten Teile „Nominalergänzung“ und „Adjektivalergänzung“ entfernt und der Teil „Modifikativergänzung“ hinzugefügt worden. Manche Kapitel sind jetzt etwas umfangreicher (vgl. etwa 3.4.4. „Negativangaben“), es kommen auch terminologische Modifikationen vor, z.B. Modifikativangaben statt modifizierende Angaben, Stellungsregeln statt Folgeregeln. Doch wie Band 4 beruht auch dieser Band weitgehend auf der ersten Ausgabe.

An wen richtet sich die „Deutsch-polnische kontrastive Grammatik?“ Den Adressaten wird zwar nur im Vorwort der ersten Ausgabe Aufmerksamkeit geschenkt, doch die Frage ist nach wie vor aktuell. Was die Germanistikstudenten betrifft, so stellte Cirko schon vor Jahren fest: „Die selbständige Lektüre der dpg überfordert sie sprachlich und terminologisch“ (Cirko 2008:7). Angesichts der sich ständig und wohl unumkehrbar vertiefenden Misere im Schulwesen und folglich auch an den Universitäten muss man heutzutage davon ausgehen, dass sich die Studenten - vielleicht von einigen Ausnahmefällen abgesehen - dieser großen Mühe gar nicht unterziehen und dass sie damit nicht als direkte Benutzer in Frage kommen. Für Hochschullehrer, Lehrbuchautoren, (angehende) Wissenschaftler, Polonisten wie Germanisten wird aber die neue „Dpg“ als ein sehr nützliches und geschätztes Arbeitsinstrument dienen (zumal es keine andere Publikation dieser Art gibt!) und Quelle grammatischen Wissens über die beiden Sprachsysteme sein.

\section{Literatur}

CIRKo Lesław, 2008, Wissenschaftlichkeit und Anwendbarkeit auf Kollisionskurs? Warum setzt sich die $d p g$ als akademisches Lehrwerk nicht durch? in: Cirko L./Grimberg M. (Hrsg.), 2008, Zwischen Lob und Kritik: sechs Jahre Erfahrung mit der Deutsch- polnischen Grammatik (dpg). Materialien der internationalen Linguistenkonferenz, Karpacz 11. - 13.09.2006, Wrocław/ Dresden, S. 5-14.

Cirko Lesław / GrimBerg Martin (Hrsg.), 2008, Zwischen Lob und Kritik: sechs Jahre Erfahrung mit der Deutsch- polnischen Grammatik (dpg). Materialien der internationalen Linguistenkonferenz, Karpacz 11. - 13.09.2006, Wrocław/Dresden.

Cirko Lesław / Grimberg Martin / Tworek Artur (Hrsg.), 2009, DPG im Kreuzfeuer. Akten der internationalen Linguistenkonferenz, Karpacz 10. - 12.09.2007, Wrocław/Dresden.

Engel Ulrich / Rytel-Kuc Danuta / Cirko Lesław / DęBSKi Antoni / GaCa Alicja / Jurasz Alina / Kątny Andrzej / Mecner Paweł / Prokop Izabela / SAdziński Roman / Schatte Christoph / Schatte Czesława / Tomiczek Eugeniusz / Weiss Daniel, 1999, Deutsch-polnische kontrastive Grammatik, Bd. 1-2, Heidelberg.

Feret Andrzej S., 2010, Zu Neuperspektiven für das Partizip in der deutsch-polnischen kontrastiven Grammatik. Kritisch zur Beschreibung der Partizipien in der Dpg, in: Studia Germanica Resoviensia 8, S. 141-147.

LiPCZuK Ryszard, 2000, [Rez.] Deutsch-polnische kontrastive Grammatik, in: Glottodidactica 28, S. $196-199$.

NAGÓRко Alicja, 2000, [Rez.] Deutsch-polnische kontrastive Grammatik, in: Biuletyn Polskiego Towarzystwa Językoznawczego 56, S. 153-158.

Monika Bielińska

ORCID: 0000-0003-3450-113X

Uniwersytet Śląski w Katowicach, Polen

Studia Linguistica 38, 2019

(C) for this edition by CNS 